\title{
Targeting DNA methylation for treating triple-negative breast cancer
}

\author{
Jia Yu*,1, Jacqueline Zayas², Bo Qin ${ }^{1,3}$ \& Liewei Wang**,1 \\ ${ }^{1}$ Department of Molecular Pharmacology \& Experimental Therapeutics, Mayo Clinic, Rochester, MN 55905, USA \\ ${ }^{2}$ Mayo Clinic Graduate School of Biomedical Sciences, Mayo Clinic School of Medicine \& The Mayo Clinic Medical Scientist \\ Training Program, Mayo Clinic, Rochester, MN 55905, USA \\ ${ }^{3}$ Department of Oncology, Mayo Clinic, Rochester, MN 55905, USA \\ *Author for correspondence: Tel.: +1 507266 6205; yu.jia@mayo.edu \\ **Author for correspondence: Tel.: +1 507284 5264; wang.liewei@mayo.edu
}

Triple-negative breast cancer (TNBC) accounts for $15-20 \%$ of all invasive breast cancers and tends to have aggressive histological features and poor clinical outcomes. Unlike, estrogen receptor- or HER2-positive diseases, TNBC patients currently lack the US FDA-approved targeted therapies. DNA methylation is a critical mechanism of epigenetic modification. It is well known that aberrant DNA methylation contributes to the malignant transformation of cells by silencing critical tumor suppressor genes. DNA methyltransferase inhibitors reactivate silenced tumor suppressor genes and result in tumor growth arrest, with therapeutic effects observed in patients with hematologic malignancies. The antitumor effect of these DNA methyltransferase inhibitors has also been explored in solid tumors, especially in TNBC that currently lacks targeted therapies.

First draft submitted: 30 May 2019; Accepted for publication: 23 August 2019; Published online: 22 November 2019

Keywords: breast cancer $\bullet$ DNA methylation $\bullet$ DNA methyltransferase $\bullet$ DNA methyltransferase inhibitor $\bullet$ TNBC

Breast cancer is the second leading cause of cancer death in women in the USA, with 41,760 women predicted to die from this disease in 2019 [1]. Triple-negative breast cancer (TNBC) defines a subtype of breast cancer that does not, or minimally expresses estrogen receptor (ER), progesterone receptor (PR) and HER2 [2]. TNBC is an aggressive disease that occurs in approximately $15-20 \%$ of all invasive breast cancers and it is often diagnosed in younger patients [3]. TNBC patients do not benefit from endocrine therapy or trastuzumab since their tumors do not express the drug targets of ER or HER2, respectively. Chemotherapy is currently the mainstay of systemic medical treatment. Although many patients with TNBC respond well to standard taxane and anthracycline-based chemotherapy, the long-term outcome is very poor due to high rates of relapse and disease recurrence $[4,5]$. The identification and application of new agents against TNBC breast cancer are an area of active research.

DNA methylation is one of the best described epigenetic events that regulate chromatin compaction and gene expression. Cancer cells utilize DNA methylation as a strategy to abnormally silence a variety of genes, including tumor suppressors and genes controlling the drug sensitivity [6]. The inhibition of DNA methylation with cytidine analogs such as 5-azacytidine (Vidaza ${ }^{\circledR}$ ) or its deoxy derivative decitabine (5-aza-2'-deoxycytidine, Dacogen ${ }^{\circledR}$ ) reactivates the expression of genes silenced by hypermethylation, reduces the malignant cell burden and improves patient survival $[7,8]$. This led to the US FDA approval of the DNA methyltransferase (DNMT) inhibitors for the treatment of hematological malignancies, and has become the standard of care for patients with high-risk myelodysplastic syndrome (MDS) [9,10].

\section{DNA methylation in TNBC}

DNA methylation is a reversible process of adding a methyl group to the fifth position of the pyrimidine ring of cytosine residues to form 5-methylcytosine (5-mC). This commonly occurs at $\mathrm{CpG}$ sites within the genome [11,12]. DNA methylation is a heritable epigenetic process regulating gene function in normal mammalian development $[13,14]$. However, aberrant methylation of $\mathrm{CpG}$ islands in the promoter regions of genes critical for cell growth is a hallmark of many cancers $[15,16]$. The phenomenon of regional DNA hypermethylation and silencing of tumor suppressor 
genes in cancer has drawn the attention of researchers and clinicians for the last decade. Recent whole-genome approaches have identified breast cancer-specific DNA methylation signatures that were correlated with cancer stage and this finding will extend our ability to classify breast cancer to better predict clinical outcome beyond what is currently possible [17-19].

The Cancer Genome Atlas Network and several other groups have demonstrated that TNBC tumors are characterized by the most extensive hypomethylation whereas ER-positive breast cancers show the highest degree of hypermethylation [20-23]. Moreover, TNBC have a distinct methylation pattern. A recent comprehensive TNBC methylome analysis by Stirzaker et al. found that TNBC patients can be stratified into three methylation clusters. TNBC patients with a hypomethylation profile were associated with a better 5 -year survival compared with clusters with heavier methylation. They also identified a series of differentially methylated regions that can stratify TNBC patients into a better or worse prognosis [24]. By comparing the methylation pattern in human embryonic stem cells, cancer cell lines, as well as human primary tissue, studies showed that the CpG sites in TNBC tumors mostly were not hypermethylated, similar to human embryonic stem cells and normal breast tissues. However, in the rest of the breast cancer subtypes, these sites were hypermethylated [25].

Interestingly, a hypermethylation phenotype of specific genes has also been described for TNBC despite global

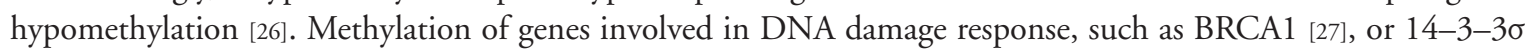
(also known as HME1) [28], has been described in TNBC. Methylation of the BRCA1 promoter is associated

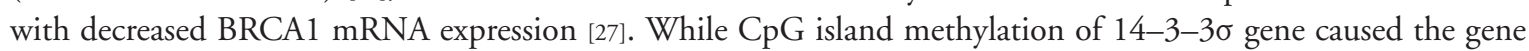
expression reduction and this mechanism was demonstrated both in cell line-based and tissue-based studies [29]. Cell-to-cell adhesion molecules such as E-cadherin, whose silencing might promote metastasis were also found to be methylated in TNBC [30]. In addition, DNA methylation in genes involving in stem cell properties changed in TNBC primary breast cancer samples and was found to be correlated with TNBC subtype and clinically aggressive phenotype [31,32]. Recently, Coyle et al. discovered over 1400 CpG sites with differential DNA methylation status between all-trans retinoic acid-sensitive and -resistant TNBC cell lines. And they further found that these sites methylation status can predict TNBC patient-derived xenograft (PDX) response to all-trans retinoic acid [33].

\section{DNA methyltransferases normal function}

In human cells, DNA methylation is mainly catalyzed by the DNA cytosine methyltransferases family members including DNMT1, DNMT3A and DNMT3B [34]. DNMT1 is the most abundant form of DNA methyltransferase and is responsible for the maintenance of methylation pattern during DNA replication [35,36. DNMT1 methylates newly synthesized DNA strands to effectively reinstate the methylation patterns that originated in the parental strands. DNMT3A and DNMT3B are the de novo DNA methyltransferases, which are responsible for de novo DNA methylation during early development and gametogenesis [37]. DNMT3L is another member of the DNMT3 family proteins and encodes a catalytically inactive protein. DNMT3L does not bind to DNA, but instead binds to DNMT3A/B and strikingly increases their catalytic activity [38]. The maintenance versus de novo function of these enzymes is not mutually exclusive. For example, DNMT1 can function as a de novo DNMT, and overexpression of DNMT1 leads to de novo methylation of CpG islands [39], and human cancer cells lacking DNMT1 have shown only a $20 \%$ reduction in methylation of CpG sties [40]. Similarly, DNMT3A or DNMT3B can function as maintenance DNMTs [40].

\section{DNA methyltransferases in TNBC DNMT1}

DNMT1 is overexpressed in many types of cancers, including breast cancer, and the expression of DNMT1 was found to be higher in TNBC and lower in luminal samples [41]. Overexpression of DNMT1 is associated with cellular transformation while reduced DNMT1 expression seems to be associated with a protective effect $[42,43]$. DNMT1 was also observed to be upregulated in most cancer-associated fibroblasts relative to their adjacent normal fibroblasts and enhanced cancer-associated fibroblasts tumor-promoting properties [44].

\section{DNMT3A/B}

DNMT3A and DNMT3B are also reported to be overexpressed in cancer tissues [35]. DNMT3B overexpression contributes to a hypermethylator phenotype in human breast cancer cell lines [45]. DNMT3B overexpression is associated with increased DNMT activity and corresponds to high rates of methylation-dependent gene silencing compared with low-frequency methylator cells. DNMT3B overexpression is found associated with TNBC and 
displayed increased proliferation and poor patient prognosis [46]. These findings are in agreement with our recent study revealing that DNMT inhibitors might be more effective in treating TNBCs overexpressing DNMT3B [47]. Additionally, transcriptional induction of DNMTs, including DNMT3A, has also been reported in cancer [48,49]. Mutations in DNMT3a are also associated with poor overall survival in acute myeloid leukemia (AML), leading to potentiation of aberrant stemness genes linked to AML development [50].

\section{DNA methyltransferase inhibitors}

With the understanding of how DNA methylation can drive cancer, there is an increasing focus on developing pharmacological interventions for treatment of cancer. Currently, two DNMT inhibitors (DNMTi) have been approved by the FDA: azacitidine (Vidaza; Celgene) and decitabine (5 aza 2' deoxycytidine; Dacogen; SuperGen) for the treatment of patients with AML and MDS, respectively [9]. These nucleoside analogs were originally designed as cytotoxic agents with high dose. However, patients experienced severe adverse side effects. With improved understanding of their mechanism of action, low dose DNMTi (nanomolar range) is being used to achieve effective inhibition of DNA methylation in patients while also improving the tolerability [51]. Another demethylating agent in the family of nucleoside analogs is zebularine, which has been characterized as a potent, chemically stable and better bioavailable oral compound. It has promising in vitro results, encouraging zebularine use for future clinical trials [52,53].

The next-generation DNMTi includes guadecitabine (SGI-110), a prodrug whose active metabolite is decitabine. This compound has a novel molecular structure, which makes it resistant to degradation by the enzyme, cytidine deaminase and thereby prolongs in vivo exposure, increasing its efficacy [54]. The Phase II clinical trials for the treatment of AML cohorts showed promising activity [55]. Recent Phase II trial for treating MDS has indicated that guadecitabine may improve the responses and overall survival in patients who progressed on previous demethylating agents, offering a new therapeutic option for patients who might fail on currently available demethylating agents [56]. Additional demethylating agents have been explored and reviewed previously [57].

Early Phase I and II trials investigating the role of demethylating agents in solid tumors, including breast cancer, yielded little success $[58,59]$. TNBC tumors do not express ER or HER2, and they are not amenable to the conventional targeted therapies. DNMTi can induce the re-expression of endogenous ER $\alpha$ and PRs in TNBC cells $[60,61]$. Therefore, a Phase II study was conducted to evaluate the efficacy of hormonal therapy following epigenetic therapy in TNBCs [62]. Although the results from using DNMTi alone in treating cancer are modest, DNMTi in combination with histone deacetylase (HDAC) inhibitors have shown robust efficacy in clinical trials $[63,64]$.

\section{Conclusion}

Despite the extensive studies regarding the role of DNA methylation in TNBC, significant challenges remain in terms of successful application of demethylating agents in the clinic. With the recent advances in 'omics', future identification of effective biomarkers will enhance our ability to use these agents in a most appropriate way for any individual patient. With the success of developing TNBC PDX models [65,66], patient-derived organoid models $[67,68]$ and $3 \mathrm{D}$ high-throughput screening platforms [69], preclinical studies can be conducted to test related hypotheses and validate in vitro findings. Robust preclinical and translational studies are essential to support the development of new regimes and to maximize the chance of success in treating TNBCs.

\section{Future perspective}

Alterations in DNA methylation are frequently detected in TNBCs, hence agents that target these alterations are of great interest and are under investigation. Besides developing new DNMTis with higher potency, new strategies of combination therapies, targeting specific processes involved in tumorigenesis, or identifying new biomarkers predicting treatment response are required to allow achieving optimal therapeutic effect of epigenetic therapies in TNBC.

Aberrant promoter methylation of key genes may cause gene expression silencing and contribute to tumor cell spread and metastasis, thus, DNMTi can reverse the process and inhibit cancer metastasis [70]. Su et al. also found that TNBC was sensitive to DNMTi treatment and that DNMTi exerts antitumor activity, in part, by epigenetically reprogramming epithelial-to-mesenchymal transition [71]. DNMTi may provide extended value in inhibiting epithelial-to-mesenchymal transition and cancer metastasis. 
Cancer stem cells (CSCs) are found within tumors and they possess self-renewal and tumorigenic characteristics. CSCs can survive chemotherapies and cause cancer relapse. Pathania et al. found that DNMT1 was required for the maintenance of cancer stem cell in breast cancer, and inhibition of DNMT activity in breast cancer reduces cell growth, migration and cancer stem cell formation [72], suggesting that DNMTis may target the CSCs.

A recent study identified that major histocompatibility class-I genes were methylated in human breast cancers and their expression were suppressed. The DNMT inhibitor, guadecitabine, could upregulate major histocompatibility class-I in breast cancer cells, which promoted recruitment of $\mathrm{CD}^{+} \mathrm{T}$ cells to the microenvironment [73]. This immunomodulatory effect of demethylating agents may be useful to potentiate antitumor immunity and responses to checkpoint inhibition in immune-refractory breast cancers.

Although DNMTis are widely used in treating AML and MDS, the response rate in patients is around $50 \%[7]$. Given the heterogeneity of TNBC, identification of predictive biomarkers is very crucial for successful application of these drugs in clinic. DNMT3A has been associated with CpGs hypermethylation in AML [74]. Moreover, the expression levels of DNMT3A and DNMT3B are also shown to be associated with decitabine sensitivity in embryonic cells [75]. Furthermore, DNMT3A/DNMT3B double-null embryonic stem cells are more resistant to decitabine treatment than DNMT1 null cells, suggesting that decitabine may be more effective for selected types of cancer cells in which DNMT3 expression is upregulated. We also found that DNMT protein levels, especially DNMT3A and DNMT3B, can be predictive of decitabine treatment response in TNBC cells and PDX models [47]. This accumulation of evidence suggests that DNMT3A/3B levels might be the biomarkers predictive of decitabine response. We also found that decitabine could induce ubiquitin-dependent degradation of DNMTs, raising a possibility of using DNMT as pharmacodynamics biomarkers for decitabine response [47].

\section{Financial \& competing interests disclosure}

This work was supported by the Mayo Clinic Center for Individualized Medicine; the NIH (RO1 CA 196648 to L Wang). The authors have no other relevant affiliations or financial involvement with any organization or entity with a financial interest in or financial conflict with the subject matter or materials discussed in the manuscript apart from those disclosed.

No writing assistance was utilized in the production of this manuscript.

\section{Open access}

This work is licensed under the Attribution-NonCommercial-NoDerivatives 4.0 Unported License. To view a copy of this license, visit http://creativecommons.org/licenses/by-nc-nd/4.0

\section{References}

1. US breast cancer statistics (2019). www.breastcancer.org/symptoms/understand_bc/statistics

2. Dent R, Trudeau M, Pritchard KI et al. Triple-negative breast cancer: clinical features and patterns of recurrence. Clin. Cancer Res. 13(15 Pt 1), 4429-4434 (2007).

3. Trivers KF, Lund MJ, Porter PL et al. The epidemiology of triple-negative breast cancer, including race. Cancer Causes Control 20(7), 1071-1082 (2009).

4. Liedtke C, Mazouni C, Hess KR et al. Response to neoadjuvant therapy and long-term survival in patients with triple-negative breast cancer. J. Clin. Oncol. 26(8), 1275-1281 (2008).

5. Gluz O, Liedtke C, Gottschalk N, Pusztai L, Nitz U, Harbeck N. Triple-negative breast cancer - current status and future directions. Ann. Oncol. 20(12), 1913-1927 (2009).

6. Esteller M, Corn PG, Baylin SB, Herman JG. A gene hypermethylation profile of human cancer. Cancer Res. 61(8), 3225-3229 (2001).

7. Fenaux P, Mufti GJ, Hellstrom-Lindberg E et al. Efficacy of azacitidine compared with that of conventional care regimens in the treatment of higher-risk myelodysplastic syndromes: a randomised, open-label, Phase III study. Lancet Oncol. 10(3), 223-232 (2009).

8. Lubbert M, Suciu S, Hagemeijer A et al. Decitabine improves progression-free survival in older high-risk MDS patients with multiple autosomal monosomies: results of a subgroup analysis of the randomized Phase III study 06011 of the EORTC Leukemia Cooperative Group and German MDS Study Group. Ann. Hematol. 95(2), 191-199 (2016).

9. Kaminskas E, Farrell A, Abraham S et al. Approval summary: azacitidine for treatment of myelodysplastic syndrome subtypes. Clin. Cancer Res. 11(10), 3604-3608 (2005).

10. Silverman LR. DNA methyltransferase inhibitors in myelodysplastic syndrome. Best Pract. Res. Clin. Haematol. 17(4), 585-594 (2004).

11. Law JA, Jacobsen SE. Establishing, maintaining and modifying DNA methylation patterns in plants and animals. Nat. Rev. Genet. 11(3), 204-220 (2010).

12. Jones PA. Functions of DNA methylation: islands, start sites, gene bodies and beyond. Nat. Rev. Genet. 13(7), 484-492 (2012). 
13. Compere SJ, Palmiter RD. DNA methylation controls the inducibility of the mouse metallothionein-I gene lymphoid cells. Cell 25(1), 233-240 (1981).

14. Holliday R, Pugh JE. DNA modification mechanisms and gene activity during development. Science 187(4173), 226-232 (1975).

15. Sharma S, Kelly TK, Jones PA. Epigenetics in cancer. Carcinogenesis 31(1), 27-36 (2010).

16. Ellis L, Atadja PW, Johnstone RW. Epigenetics in cancer: targeting chromatin modifications. Mol. Cancer Ther. 8(6), 1409-1420 (2009).

17. Szyf M. DNA methylation signatures for breast cancer classification and prognosis. Genome Med. 4(3), 26 (2012).

18. Dedeurwaerder S, Desmedt C, Calonne E. Largest ever DNA methylation dataset for breast cancer completed. Expert Rev. Mol. Diagn. 11(5), 470 (2011).

19. Flanagan JM, Cocciardi S, Waddell $\mathrm{N}$ et al. DNA methylome of familial breast cancer identifies distinct profiles defined by mutation status. Am. J. Hum. Genet. 86(3), 420-433 (2010).

20. Roll JD, Rivenbark AG, Sandhu R et al. Dysregulation of the epigenome in triple-negative breast cancers: basal-like and claudin-low breast cancers express aberrant DNA hypermethylation. Exp. Mol. Pathol. 95(3), 276-287 (2013).

21. Stefansson OA, Moran S, Gomez A et al. A DNA methylation-based definition of biologically distinct breast cancer subtypes. Mol. Oncol. 9(3), 555-568 (2015).

22. Koboldt DC, Fulton RS, McLellan MD et al. Comprehensive molecular portraits of human breast tumours. Nature 490(7418), 61-70 (2012).

23. Gao Y, Jones A, Fasching PA et al. The integrative epigenomic-transcriptomic landscape of ER positive breast cancer. Clin. Epigenetics 7(1), 126 (2015).

24. Stirzaker C, Zotenko E, Song JZ et al. Methylome sequencing in triple-negative breast cancer reveals distinct methylation clusters with prognostic value. Nat. Commun. 6, 5899 (2015).

25. Calvanese V, Horrillo A, Hmadcha A et al. Cancer genes hypermethylated in human embryonic stem cells. PLoS ONE 3(9), e3294 (2008).

26. Sandhu R, Rivenbark AG, Coleman WB. Enhancement of chemotherapeutic efficacy in hypermethylator breast cancer cells through targeted and pharmacologic inhibition of DNMT3b. Breast Cancer Res. Treat. 131(2), 385-399 (2012).

27. Rice JC, Ozcelik H, Maxeiner P, Andrulis I, Futscher BW. Methylation of the BRCA1 promoter is associated with decreased BRCA1 mRNA levels in clinical breast cancer specimens. Carcinogenesis 21(9), 1761-1765 (2000).

28. Ferguson AT, Evron E, Umbricht CB et al. High frequency of hypermethylation at the 14-3-3 sigma locus leads to gene silencing in breast cancer. Proc. Natl Acad. Sci. USA 97(11), 6049-6054 (2000).

29. Yang X, Yan L, Davidson NE. DNA methylation in breast cancer. Endocr. Relat. Cancer 8(2), 115-127 (2001).

30. Kashiwagi S, Yashiro M, Takashima T et al. Significance of E-cadherin expression in triple-negative breast cancer. Br. J. Cancer 103(2), 249-255 (2010).

31. Kagara N, Huynh KT, Kuo C et al. Epigenetic regulation of cancer stem cell genes in triple-negative breast cancer. Am. J. Pathol. 181(1), 257-267 (2012).

32. Temian DC, Pop LA, Irimie AI, Berindan-Neagoe I. The epigenetics of triple-negative and basal-like breast cancer: current knowledge. J. Breast Cancer 21(3), 233-243 (2018).

33. Coyle KM, Dean CA, Thomas ML et al. DNA methylation predicts the response of triple-negative breast cancers to all-trans retinoic acid. Cancers (Basel) 10(11), 397 (2018)

34. Jeltsch A. Molecular enzymology of mammalian DNA methyltransferases. Curr. Top. Microbiol. Immunol. 301, 203-225 (2006).

35. Robertson KD, Uzvolgyi E, Liang G et al. The human DNA methyltransferases (DNMTs) 1, 3a and 3b: coordinate mRNA expression in normal tissues and overexpression in tumors. Nucleic Acids Res. 27(11), 2291-2298 (1999).

36. Kar S, Deb M, Sengupta D et al. An insight into the various regulatory mechanisms modulating human DNA methyltransferase 1 stability and function. Epigenetics 7(9), 994-1007 (2012).

37. Okano M, Bell DW, Haber DA, Li E. DNA methyltransferases Dnmt3a and Dnmt3b are essential for de novo methylation and mammalian development. Cell 99(3), 247-257 (1999).

38. Gowher H, Liebert K, Hermann A, Xu GL, Jeltsch A. Mechanism of stimulation of catalytic activity of Dnmt3A and Dnmt3B DNA-(cytosine-C5)-methyltransferases by Dnmt3L. J. Biol. Chem. 280(14), 13341-13348 (2005).

39. Vertino PM, Yen RWC, Gao J, Baylin SB. De novo methylation of CpG island sequences in human fibroblasts overexpressing DNA (cytosine-5)-methyltransferase. Mol. Cell. Biol. 16(8), 4555-4565 (1996).

40. Rhee I, Jair KW, Yen RWC et al. CpG methylation is maintained in human cancer cells lacking DNMT1. Nature 404(6781), 1003-1007 (2000).

41. Shin E, Lee Y, Koo JS. Differential expression of the epigenetic methylation-related protein DNMT1 by breast cancer molecular subtype and stromal histology. J. Transl. Med. 14, 87 (2016). 
42. Wu J, Issa JP, Herman J, Bassett DE Jr, Nelkin BD, Baylin SB. Expression of an exogenous eukaryotic DNA methyltransferase gene induces transformation of NIH 3T3 cells. Proc. Natl Acad. Sci. USA 90(19), 8891-8895 (1993).

43. Belinsky SA, Nikula KJ, Baylin SB, Issa JPJ. Increased cytosine DNA-methyltransferase activity is target-cell-specific and an early event in lung cancer. Proc. Natl Acad. Sci. USA 93(9), 4045-4050 (1996).

44. Al-Kharashi LA, Al-Mohanna FH, Tulbah A, Aboussekhra A. The DNA methyl-transferase protein DNMT1 enhances tumor-promoting properties of breast stromal fibroblasts. Oncotarget 9(2), 2329-2343 (2018).

45. Roll JD, Rivenbark AG, Jones WD, Coleman WB. DNMT3b overexpression contributes to a hypermethylator phenotype in human breast cancer cell lines. Mol. Cancer 7, 15 (2008).

46. Girault I, Tozlu S, Lidereau R, Bieche I. Expression analysis of DNA methyltransferases 1, 3A, and 3B in sporadic breast carcinomas. Clin. Cancer Res. 9(12), 4415-4422 (2003).

47. Yu J, Qin B, Moyer AM et al. DNA methyltransferase expression in triple-negative breast cancer predicts sensitivity to decitabine. J. Clin. Invest. 128(6), 2376-2388 (2018).

48. Bakin AV, Curran T. Role of DNA 5-methylcytosine transferase in cell transformation by fos. Science 283(5400), 387-390 (1999).

49. Jinawath A, Miyake S, Yanagisawa Y, Akiyama Y, Yuasa Y. Transcriptional regulation of the human DNA methyltransferase 3A and 3B genes by Sp3 and Sp1 zinc finger proteins. Biochem. J. 385(Pt 2), 557-564 (2005).

50. Lu R, Wang P, Parton T et al. Epigenetic perturbations by Arg882-mutated DNMT3A potentiate aberrant stem cell gene-expression program and acute leukemia development. Cancer Cell 30(1), 92-107 (2016).

51. Issa JP. Optimizing therapy with methylation inhibitors in myelodysplastic syndromes: dose, duration, and patient selection. Nat. Clin. Pract. Oncol. 2(Suppl. 1), S24-S29 (2005).

52. Ben-Kasus T, Ben-Zvi Z, Marquez VE, Kelley JA, Agbaria R. Metabolic activation of zebularine, a novel DNA methylation inhibitor, in human bladder carcinoma cells. Biochem. Pharmacol. 70(1), 121-133 (2005).

53. Cheng JC, Matsen CB, Gonzales FA et al. Inhibition of DNA methylation and reactivation of silenced genes by zebularine. J. Natl Cancer Inst. 95(5), 399-409 (2003).

54. Chuang JC, Warner SL, Vollmer D et al. S110, a 5-aza-2'-deoxycytidine-containing dinucleotide, is an effective DNA methylation inhibitor in vivo and can reduce tumor growth. Mol. Cancer Ther. 9(5), 1443-1450 (2010).

55. Kantarjian HM, Roboz GJ, Kropf PL et al. Guadecitabine (SGI-110) in treatment-naive patients with acute myeloid leukaemia: Phase II results from a multicentre, randomised, Phase I/II trial. Lancet Oncol. 18(10), 1317-1326 (2017).

56. Garcia-Manero G, Roboz G, Walsh K et al. Guadecitabine (SGI-110) in patients with intermediate or high-risk myelodysplastic syndromes: Phase II results from a multicentre, open-label, randomised, Phase I/II trial. Lancet Haematol. 6(6), 317-327 (2019).

57. Gros C, Fahy J, Halby L et al. DNA methylation inhibitors in cancer: recent and future approaches. Biochimie 94(11), $2280-2296$ (2012).

58. Weiss AJ, Stambaugh JE, Mastrangelo MJ, Laucius JF, Bellet RE. Phase I study of 5-azacytidine (NSC-102816). Cancer Chemother. Rep. 56(3), 413-419 (1972).

59. Weiss AJ, Metter GE, Nealon TF et al. Phase II study of 5-azacytidine in solid tumors. Cancer Treat. Rep. 61(1), 55-58 (1977).

60. Li Y, Yuan YY, Meeran SM, Tollefsbol TO. Synergistic epigenetic reactivation of estrogen receptor- $\alpha$ (ER $\alpha$ ) by combined green tea polyphenol and histone deacetylase inhibitor in ER $\alpha$-negative breast cancer cells. Mol. Cancer 9, 274 (2010).

61. Gajulapalli VNR, Malisetty VL, Chitta SK, Manavathi B. Oestrogen receptor negativity in breast cancer: a cause or consequence? Biosci. Rep. 36(6), e00432 (2016).

62. Connolly RM, Jankowitz RC, Andreopoulou E et al. A Phase II study investigating the safety, efficacy and surrogate biomarkers of response of 5-azacitidine (5-AZA) and entinostat (MS-275) in patients with advanced breast cancer. Cancer Res. 71(suppl. 24), Abstract nr OT3-01-06 (2011).

63. Cameron EE, Bachman KE, Myohanen S, Herman JG, Baylin SB. Synergy of demethylation and histone deacetylase inhibition in the re-expression of genes silenced in cancer. Nat. Genet. 21(1), 103-107 (1999).

64. Griffiths EA, Gore SD. DNA methyltransferase and histone deacetylase inhibitors in the treatment of myelodysplastic syndromes. Semin. Hematol. 45(1), 23-30 (2008).

65. Yu J, Qin B, Moyer AM et al. Establishing and characterizing patient-derived xenografts using pre-chemotherapy percutaneous biopsy and post-chemotherapy surgical samples from a prospective neoadjuvant breast cancer study. Breast Cancer Res. 19(1), 130 (2017).

66. Townsend EC, Murakami MA, Christodoulou A et al. The public repository of xenografts enables discovery and randomized Phase II-like trials in mice. Cancer Cell 29(4), 574-586 (2016).

67. Sachs N, de Ligt J, Kopper O et al. A living biobank of breast cancer organoids captures disease heterogeneity. Cell 172(1-2), 373.e10-386.e10 (2018).

68. Drost J, Clevers H. Organoids in cancer research. Nat. Rev. Cancer 18(7), 407-418 (2018). 
69. Lanz HL, Saleh A, Kramer B et al. Therapy response testing of breast cancer in a 3D high-throughput perfused microfluidic platform. BMC Cancer 17(1), 709 (2017).

70. Borges S, Doppler H, Perez EA et al. Pharmacologic reversion of epigenetic silencing of the PRKD1 promoter blocks breast tumor cell invasion and metastasis. Breast Cancer Res. 15(2), R66 (2013).

71. Su Y, Hopfinger NR, Nguyen TD, Pogash TJ, Santucci-Pereira J, Russo J. Epigenetic reprogramming of epithelial mesenchymal transition in triple negative breast cancer cells with DNA methyltransferase and histone deacetylase inhibitors. J. Exp. Clin. Cancer Res. 37(1), 314 (2018).

72. Pathania R, Ramachandran S, Elangovan $S$ et al. DNMT1 is essential for mammary and cancer stem cell maintenance and tumorigenesis. Nat. Commun. 6, 6910 (2015).

73. Luo N, Nixon MJ, Gonzalez-Ericsson PI et al. DNA methyltransferase inhibition upregulates MHC-I to potentiate cytotoxic T lymphocyte responses in breast cancer. Nat. Commun. 9(1), 248 (2018).

74. Spencer DH, Russler-Germain DA, Ketkar $S$ et al. CpG island hypermethylation mediated by DNMT3A is a consequence of AML progression. Cell 168(5), 801-816 (2017).

75. Oka M, Meacham AM, Hamazaki T, Rodic N, Chang LJ, Terada N. De novo DNA methyltransferases Dnmt3a and Dnmt3b primarily mediate the cytotoxic effect of 5-aza-2'-deoxycytidine. Oncogene 24(19), 3091-3099 (2005). 
